\title{
INVESTIGACIONES
}

\section{Uso de las TIC en el hogar: Entre el entretenimiento y el aprendizaje informal}

\author{
Use of ICT at home: Between entertainment and informal learning
}

\begin{abstract}
Carolina Matamala Riquelme
Becaria Conicyt. Programa Becas Chile para estudios de Doctorado en el Extranjero Doctoranda de sociología y antropología en la Universidad Complutense de Madrid. Telf.: (34)678620562. Correo electrónico: caromata@ucm.es
\end{abstract}

\begin{abstract}
RESUMEN
El siguiente artículo tiene como objetivo describir los impactos y aprendizajes derivados del uso de las TIC en el hogar. En la investigación se utilizó una metodología cualitativa basada en el uso de entrevistas y blogs a una muestra intencionada de estudiantes españoles de secundaria. De acuerdo a los resultados de la investigación, se pueden establecer cuatro tipos de impactos. Entre los impactos positivos se puede observar que las TIC mejoran los procesos educativos, por un lado, y facilitan las actividades de recreación de los estudiantes, por otro, logrando apoyar procesos informales de aprendizaje. En lo que respecta a los impactos negativos, se identifica, por un lado, la interferencia de las TIC en los procesos de estudio y, por otro, se advierte una serie de riesgos asociados al mal uso de las tecnologías.
\end{abstract}

Palabras Claves: nativos digitales, uso de las TIC, impacto de uso de las TIC, aprendizaje informal.

\section{ABSTRACT}

The following article aims to describe the impacts and lessons learned from the use of ICT at home. A qualitative methodology based on the use of interviews and blogs to a purposive sample of Spanish secondary school students was used in the investigation. According to research results, we can establish four types of impacts. Among the positive impacts, we can see that ICT improves the educational process on the one hand and facilitate the recreation of the students on the other; thus supporting informal learning processes. With regard to the negative impacts identified, on the one hand interference of ICT in the study process and on the other, a number of risks associated with the misuse of technology are apparent.

Key words: digital natives, use of ICT, impact of ICT, informal learning. 


\section{INTRODUCCIÓN}

No cabe duda que nos encontramos en una nueva sociedad, caracterizada por la irrupción de la interconectividad y las tecnologías de información y comunicación, en la cual los jóvenes, denominados nativos digitales, parecen ser uno de los principales protagonistas dado su alto nivel de conectividad. De acuerdo a un informe de Telefónica (Reig, 2013), estos se conectan habitualmente a Internet en casi 30 puntos más que la población total, lo que ha llevado a considerarlos como un segmento experto en el uso de tecnologías de información y comunicación (en lo sucesivo TIC), con habilidades innatas que los diferencian de las generaciones anteriores (denominados inmigrantes digitales). No obstante, las investigaciones especializadas no han encontrado evidencia concluyente respecto de las habilidades en el uso de tecnología, que supuestamente caracterizan a los nativos digitales. Por el contrario, se ha constatado un uso más bien precario de las TIC, centrándose principalmente en actividades de entretenimiento y comunicación.

Por otro lado, los beneficios en términos de formación y aprendizaje que pueden obtener los estudiantes a partir de la interacción con las TIC parecen ser poco abordados. Si bien se ha realizado una serie de investigaciones respecto del impacto de las tecnologías ${ }^{1}$ en educación, estas responden principalmente a estudios experimentales que evalúan iniciativas concretas de uso de TIC en las escuelas; en este sentido, hay quienes asumen que el uso de la tecnología generará beneficios per se, sin evidencias empíricas concretas, con lo cual el estudio respecto de cómo interactúan los jóvenes con las tecnologías y qué beneficios pueden obtener de ellas, parece ser un flanco abierto de investigación, principalmente en lo referido al impacto del uso informal de las TIC (Livingstone, 2012; Ruiz, 2013; Selwyn, 1998).

En coherencia, en el artículo se busca dar respuesta a los siguientes objetivos de investigación:

1. Establecer cuáles son los impactos del uso de las TIC en el hogar que perciben los estudiantes.

2. Establecer cuáles son los aprendizajes que logran los estudiantes a través del uso informal de las TIC en el hogar.

\section{MARCO CONCEPTUAL}

\subsection{USO DE LAS TIC DE LOS NATIVOS DIGITALES}

En los últimos años se ha observado un aumento de usuarios de las tecnologías de información y comunicación, lo cual es particularmente evidente entre adolescentes y jóvenes (García y Rosado, 2012), quienes comienzan a utilizar las tecnologías a los nueve años promedio. De acuerdo a estudios realizados en el contexto europeo, se estableció que un $60 \%$ de adolescentes y jóvenes acceden a lo menos una vez al día a Internet, utilizando las TIC una hora y media en promedio. El $87 \%$ accede a las TIC desde su hogar y un $63 \%$ desde la escuela. Solo un $12 \%$, accede desde lugares públicos como locutorios (cibercafé) o bibliotecas (Livingstone et al., 2011); resultados que son coincidentes con

Se hablará indistintamente de TIC y tecnologías. 
otras investigaciones en España (Espinar y González, 2008; Gordo, 2006) y Latinoamérica (Pavez, 2014; Sunkel, Trucco y Moller, 2011).

En este contexto, la idea de una nueva generación adepta a la tecnología ha tomado fuerza, particularmente desde que surgieron conceptos como nativos digitales (Prensky, 2001) Generación milenaria (Howe y Strauss, 2000; Oblinger, 2003), Generación gamer (Carstens y Beck, 2005) o Aprendices del nuevo milenio (Pedró, 2006), enfatizando en las diferencias generacionales y en las habilidades tecnológicas y personales de quienes nacieron posterior a los 80 .

$\mathrm{Al}$ respecto, se han generado dos posturas bien diferenciadas (Cabra y Marciales, 2009; Robert, 2013; Sánchez et al., 2011). La primera representa a los jóvenes como una generación multitarea, capaz de trabajar en red, aprender lúdicamente, crear sus propios contenidos y ser creativos con la tecnología; destacando su preferencia por la inmediatez y los accesos al azar (Bruns, 2007; García et al., 2007; Hull, 2011; Oblinger, 2003; Tapscott, 1998; Yong y Gates, 2014). La segunda postura afirma que no existen estudios rigurosos, con muestras representativas, que den cuenta de las habilidades y características de los nativos digitales, sino, por el contrario, dichas afirmaciones se basarían en observaciones informales, anécdotas o creencias a partir del sentido común (Bennett, Maton y Kervin, 2008; Selwyn, 2009). En este sentido, el concepto de nativo digital, sería discursivo y no descriptivo (Keen, 2007).

En efecto, diversos estudios coinciden en señalar que los nativos digitales hacen un uso limitado de las tecnologías (García, López-de-Ayala y Catalina, 2013; Kennedy et al., 2008; Kennedy et al., 2010; Selwyn, 2009; Thompson, 2013), predominando los juegos, actividades de comunicación y búsqueda de información (Heim et al., 2007; Hinostroza et al., 2014; Peter y Valkenburg, 2006; Ruiz, 2013).

De acuerdo a Yong y Gates (2014), los jóvenes y adolescentes ocupan mayor cantidad de tiempo para navegar en Internet (3,77 horas promedio), usar el teléfono móvil (3,44 horas promedio) y escuchar música (3 horas promedio). La Internet está destinada principalmente para realizar tareas escolares, jugar, ver videos y participar de las redes sociales (Livingstone et al., 2011). Por su parte, los teléfonos móviles son utilizados principalmente para recibir y entregar contenidos, ver videos, jugar y navegar por Internet, aumentando la mensajería instantánea y disminuyendo el uso de correo electrónico (Williams et al., 2012).

Solo una minoría de jóvenes y adolescentes se dedican a la creación de sus propios contenidos multimedia para la Web, y solo un $25 \%$ de los jóvenes utiliza tecnologías emergentes (Bennett et al., 2008). No obstante, se observa un alto porcentaje de adolescentes y jóvenes que utilizan tecnologías para estudiar o realizar trabajos escolares (Oliver y Goerke, 2007).

\subsection{USO E IMPACTO DE LAS TIC EN EDUCACIÓN}

Las TIC han penetrado fuertemente en la sociedad y en los sistemas educativos de forma particular, con la promesa de mejorar la educación, por un lado, y convertirse en un catalizador para el cambio educativo, por otro. En coherencia, los centros educativos han sido dotados de infraestructura tecnológica, presentando avances importantes en las últimas décadas e implementándose una serie de programas para su funcionamiento pedagógico.

Respecto de evidencias que demuestren el impacto de las TIC en el aprendizaje de los estudiantes, destacan los meta análisis de Kulik (2003), quien constató el aporte del uso de las tecnologías en el mejoramiento de la escritura a través de una revisión de 400 estudios. Resultados similares a los hallados por Balanskat y Blamire (2007), quienes confirmaron 
un mejor rendimiento en matemáticas, lenguajes y ciencias a nivel primario, gracias al uso de TIC (Roman, 2010). Por su parte, el estudio ImpCT2 encontró que el procesador de texto fortalecía el desarrollo de la escritura a nivel primario (Condie y Munro, 2007), a la vez que el uso de TIC se asocia a un mejor rendimiento en matemáticas en primaria y ciencias en secundaria (Claro, 2010). Sin embargo, buena parte de los estudios que demuestran impacto en el aprendizaje están basados en la percepción del aprendizaje de profesores y alumnos y no en resultados provenientes de evaluación de contenidos (Condie y Munro, 2007; Cox y Marshall, 2007).

Por otro lado, el uso de las tecnologías de información y comunicación para actividades pedagógicas al interior del aula, sigue siendo escaso o poco frecuente, así por ejemplo, de acuerdo a datos de PISA 2009 (OECD, 2011), el porcentaje de estudiantes que utiliza TIC a lo menos 60 minutos a la semana, es inferior al 4\%. Por su parte, una serie de investigaciones (Cuban, 2003; Hinostroza et al., 2011; OECD, 2011; Pedró, 2011; RambollManagement, 2006) ha constatado que las actividades realizadas al interior de aula son de tipo tradicional, tales como buscar información o como apoyo para alguna actividad común de toda la clase. Tal como plantea Coll, Mauri y Onrubia (2008a), el profesorado tiende a utilizar las tecnologías para mantener los patrones clásicos de docencia, con lo cual se observa un efecto limitado en la transformación y mejora de las prácticas educativas. Adell y Castañeda (2010) agregan que los cursos e-learning tampoco han significado un cambio metodológico, manteniendo las formas tradicionales de enseñanza y aprendizaje.

Adicionalmente, se observan una serie de barreras relacionadas principalmente con el comportamiento de los profesores (Gu, Zhu y Guo, 2013). Así, por ejemplo, Chiappe, Mesa y Álvarez (2013) detectaron que los docentes se han acercado tímidamente al uso e implementación de tecnología tanto en clases como en su vida cotidiana. Por su parte, diversos estudios (Demetriadis et al., 2003; Ertmer et al., 1999; Gu et al., 2013; Pelgrum, 2001) han dado cuenta de cómo el nivel de confianza y seguridad de los profesores en el uso de las TIC actúa como barrera en la incorporación de tecnologías como una herramienta pedagógica. Al respecto, Roman (2010) reconoce cuatro razones empleadas por los profesores para no utilizar tecnología, i) falta de seguridad en el uso de tecnología, ii) falta de computadores suficientes para todos los alumnos, iii) preferencia por textos y materiales tradicionales y iv) falta de conocimiento técnico en el uso de tecnología. Respecto del número de computadores suficientes, Selwyn (2004) plantea que la disponibilidad de la tecnología en la sala de clases es un factor fundamental para su uso, con lo cual habrá que considerar elementos tales como calidad de la tecnología, tiempo de uso de tecnología y lugar de uso de la tecnología (sala de clases o laboratorio de informática). Pedró (2011), en cambio, sostiene que el acceso a la tecnología, si bien es importante, dado el acceso actual que existe en los centros educativos, este ha dejado de ser un elemento explicativo, otorgándole mayor importancia a la confianza que sienten los docentes en el uso de la tecnología y la importancia que estos otorgan a la tecnología como una herramienta pedagógica capaz de generar aprendizajes.

\subsection{LAS TIC COMO NUEVO ESCENARIO DE APRENDIZAJE}

Las dificultades de establecer relaciones causales más o menos directas entre el uso de las TIC e impactos de aprendizaje en un contexto educativo formal, junto al boom de la Web 2.0, han llevado a dirigir la atención a los procesos de aprendizaje informal que se producen 
fuera de las instituciones educativas con apoyo del uso de tecnologías de información y comunicación. El objetivo de mantener el interés en el potencial educativo de las TIC radica en las posibilidades que entregan estas tecnologías en el acceso y búsqueda de información, por un lado, y en la creación de entornos que integren los sistemas semióticos conocidos y amplíen la capacidad humana de procesar, transmitir y compartir conocimientos, por otro (Coll, Mauri y Onrubia, 2008b).

$\mathrm{Al}$ respecto, la Internet se ha constituido en la actualidad como la mayor fuente de información existente y uno de los entornos de aprendizaje más importante, permitiendo acceder a foros, noticias, libros, artículos científicos, videos, etc., al tiempo que conecta y comunica a un gran número de personas (Adell y Castañeda, 2010). Esta particularidad permite que los jóvenes puedan diseñar las estrategias a través de las cuales quieren aprender determinados temas o habilidades, fijándose objetivos, metas y formas de aprendizaje (Coll et al., 2014).

No obstante a lo anterior, algunos autores (Cassany y Ayala, 2008) manifiestan que los jóvenes no son conscientes de sus necesidades educativas, con lo cual no son capaces de ser autónomos en su autoformación, en este sentido no habría un impacto positivo del uso cotidiano de las TIC. Adicionalmente, algunas investigaciones han demostrado que los jóvenes realizan actividades con TIC diferenciadas en la escuela y en el hogar, toda vez que el uso de tecnologías fuera de la escuela está reservada para actividades de entretenimiento y comunicación (Ruiz, 2013).

Por el contrario, otras investigaciones han constatado un impacto positivo del acceso y uso de las TIC en el hogar. Así, por ejemplo, Chowdry, Crawford y Goodman (2009), a través de un estudio longitudinal en Inglaterra, determinaron que el acceso a computadoras e Internet se asociaba positivamente con los niveles de logro educativo. Beltran, Das y Fairlie (2008) determinaron que el uso de computadoras en el hogar aumentaba de seis a ocho puntos la probabilidad de graduarse de secundaria. Por su parte, datos de PISA demostraron asociación entre la frecuencia de uso de las TIC y el rendimiento académico; de esta forma un estudiante que hace un uso moderado de las TIC obtendrá mejor rendimiento que un estudiante que utiliza con poca o demasiada frecuencia las TIC (OECD, 2010). Por su parte, Ruiz (2013) detectó que el uso de tecnologías en el hogar está destinado en parte a prácticas colaborativas entre los jóvenes para la realización de tareas, a la vez que se identifica el desarrollo de competencias relacionadas con la creatividad, iniciativa y autonomía.

\section{METODOLOGÍA}

Para llevar a cabo la investigación, se utilizó una metodología cualitativa, orientada principalmente por las bases de la teoría fundamentada, la cual es definida por sus acuñadores como un procedimiento para generar teoría a partir de los datos mediante una investigación (Glaser y Strauss, 1974). La teoría fundamentada se plantea como una alternativa metodológica en el análisis cualitativo, que propone el desarrollo sistemático de teoría desde la conceptualización (Carrero, Soriano y Trinidad, 2012). Se trata de un procedimiento inductivo, contrario a la elaboración de teoría de manera lógica-deductiva sin un apoyo empírico (Andréu, García-Nieto y Pérez, 2007), y se encuentra sustentada especialmente en el método comparativo constante, el muestreo teórico y la saturación de categorías (Glaser y Strauss, 1974; Hood, 2010; Strauss, 1987; Strauss y Corbin, 1990; Valles, 1997). 


\subsection{PARTICIPANTES}

En particular y considerando los objetivos de esta investigación, se trabajó con una muestra de 24 estudiantes de segundo año de secundaria de centros educativos públicos y privados de Madrid (España), quienes fueron seleccionados por la frecuencia y propósitos de uso de las TIC. La selección de los estudiantes (en el marco de seis institutos seleccionados previamente) se fue desarrollando mediante las necesidades surgidas en el análisis preliminar de datos, orientando la selección hacia aquellos informantes que podían generar mayor cantidad y calidad de información (Carrero et al., 2012). De esta forma, y a raíz de las primeras entrevistas, se determinó que una de las categorías emergentes decía relación con el carácter adictivo de las TIC y su interferencia para los estudios, con lo cual se decidió recoger información respecto de estudiantes que presentaban altos y bajos niveles de uso de tecnología, conjugado con altos y bajos rendimientos académicos. Posteriormente, y de acuerdo a las nuevas categorías, se seleccionaron estudiantes según usos novedosos/tradicionales de la tecnología. La selección de estudiantes se detuvo una vez lograda la saturación teórica, entendida como el momento en el cual no surgen nuevas propiedades de las categorías estudiadas (Glaser y Strauss, 1974; Hood, 2010; Strauss, 1987; Strauss y Corbin, 1990; Valles, 1997).

\subsection{TÉCNICAS DE RECOLECCIÓN DE DATOS}

A cada estudiante seleccionado se le aplicó una entrevista en profundidad y se le solicitó que completara durante siete días un blog a través del cual debía registrar las actividades y los objetivos del uso diario de las TIC. La entrevista fue aplicada en dos momentos: en una primera instancia para generar el rapport con los informantes, explicarles los objetivos de investigación y el proceso para completar el blog. El objetivo fundamental de esta primera entrevista, siguiendo las sugerencias de Valles (2002) basado en el modelo contextual interaccionista de Gorden (1969), fue detectar los problemas de comunicación verbal que eventualmente podían generarse y confirmar las características de los entrevistados, de tal forma de determinar si podrían generar información relevante para la investigación. Durante la segunda entrevista se profundizó en los temas relevantes para la investigación, según las categorías emergentes.

El blog fue utilizado para recoger el detalle de actividades diarias con tecnología, durante una extensión de siete días, y ahondar en la experiencia de los informantes con el uso de las TIC. La aplicación de esta técnica intentó resolver un problema metodológico (la imposibilidad de la observación) y, a la vez, acercarse al uso de las tecnologías de información y comunicación como una herramienta de investigación y no solo como un objeto de estudio.

\subsection{TÉCNICAS DE ANÁLISIS DE DATOS}

Tal como se ha adelantado, el proceso de análisis se basa en las directrices de la teoría fundamentada, método que dirige su atención a la conceptualización basada en la emergencia de patrones sociales a partir de los datos recolectados en la investigación (Glaser y Strauss, 1974). Su principal estrategia de análisis es el método comparativo constante, en el cual se reconocen cuatro fases: i) Comparación de sucesos aplicables para cada categoría; ii) 
Integración de las categorías y sus propiedades, iii) Delimitación de la teoría y iv) Redacción de la teoría (Carrero et al., 2012; Glaser y Strauss, 1974; Valles, 1997).

\section{RESULTADOS}

\subsection{IMPACTO DEL USO DE LAS TIC EN EL HOGAR}

En atención al primer objetivo de investigación, se pueden establecer cuatro tipos de impactos del uso de las TIC en el hogar. La identificación de dichos impactos se realizó a partir del objetivo con que los estudiantes utilizan las TIC (educativo o personal) y los efectos que perciben de dichos usos (positivo o negativo).

Los objetivos de uso de las TIC por parte de los estudiantes entrevistados se clasifican en dos grandes áreas: una referida al área educativa, asociada a las actividades escolares realizadas por los jóvenes en su rol de estudiantes; otra referida a sus actividades personales o extraescolares, las cuales están asociadas a los pasatiempos e intereses de los estudiantes entrevistados en su rol de adolescentes o jóvenes. Por su parte, en cada una de estas áreas los informantes reconocen aspectos positivos y negativos de las TIC, según se puede apreciar en la Tabla 1.

Tabla 1. Impacto del uso de las TIC en el hogar

\begin{tabular}{|c|c|c|}
\hline & EDUCATIVOS & COTIDIANOS O PERSONALES \\
\hline \multirow{4}{*}{ POSITIVOS } & $\begin{array}{l}\text { Aprendizajes y ayuda en procesos } \\
\text { educativos }\end{array}$ & $\begin{array}{l}\text { Promoción de actividades de } \\
\text { recreación y ocio }\end{array}$ \\
\hline & $\begin{array}{l}\text { - } \quad \text { Repaso de conceptos y } \\
\text { contenidos }\end{array}$ & - Comunicación permanente \\
\hline & $\begin{array}{l}\text { Coordinación de trabajos y } \\
\text { gestión de información }\end{array}$ & $\begin{array}{l}\text { - Mayor acceso a actividades de } \\
\text { ocio }\end{array}$ \\
\hline & - $\quad$ Elaboración de productos & - $\quad$ Ayuda en el desarrollo de hobbies \\
\hline \multirow{3}{*}{ NEGATIVOS } & $\begin{array}{l}\text { Promoción de malos hábitos } \\
\text { educativos }\end{array}$ & Exposición a riesgos digitales \\
\hline & - $\quad$ Abuso del copiado y pegado & - $\quad$ Introversión digital \\
\hline & - $\quad$ Distracción en los estudios & $\begin{array}{l}\text { - } \quad \text { Riesgo de ciberbullying y } \\
\text { vulnerabilidad de datos }\end{array}$ \\
\hline
\end{tabular}

Fuente. Elaboración propia

En el caso del uso educativo, los impactos percibidos funcionan como opuestos. Por un lado, se establecen mejoras en el proceso educativo, que fortalecen el entorno individual de aprendizaje de los estudiantes, sin embargo, los estudiantes perciben que un mal uso de las TIC puede promover hábitos o procedimientos que son contraproducentes con los procesos de aprendizaje, según se detalla a continuación: 


\section{Aprendizajes y ayuda en procesos educativos}

De acuerdo a la opinión de los estudiantes, el uso de tecnologías con fines educativos los ayuda a ser más efectivos en los procesos de aprendizaje, permitiéndoles generar productos o lograr metas de forma más rápida y eficiente. Tres son las tareas en las cuales los estudiantes observan impactos positivos del uso de las TIC, a saber:

a. Repaso de conceptos y contenidos: las TIC suelen ser utilizadas como una segunda lección o un reforzamiento de lo visto en clases, puesto que los estudiantes acuden a guías, (entregadas o no por los profesores), documentales, videos tutoriales, etc., que les permiten comprender a su propio ritmo las materias vistas en clases. Agregan, además, que Internet facilita los procesos de búsqueda de información, con lo cual pueden acudir de forma más rápida a los contenidos que necesitan y acceder a significados o traducción de palabras desconocidas.

"Si te mandan un trabajo del instituto entonces tienes que hacerlo: tienes que buscar información en internet, buscar palabras que no entiendes o videos que explican lo que puedes poner en el trabajo...".

En su mayoría, estas actividades son iniciativas de los propios estudiantes, aunque eventualmente pueden ser dirigidos o estar condicionados por instrucciones entregadas por profesores. Sin embargo, es importante recalcar que este tipo de impactos es observable principalmente entre aquellos estudiantes con mayor rendimiento académico ${ }^{2}$ y capital cultural $^{3}$, distinguiéndose así brechas en la capacidad de sacar provecho de las TIC.

b. Coordinación de trabajos y gestión de información: el uso de TIC permite a los estudiantes organizar trabajos grupales a través de redes sociales. Según la información extraída de las entrevistas, el uso de grupos en redes sociales, permite enviar archivos, sistematizar información, tomar decisiones y discutir respecto de la organización de trabajos para las distintas asignaturas. Con todo, el uso de redes sociales y de tecnologías en general se concentra principalmente en organizar o gestionar trabajos grupales y no tanto para elaborarlos, observándose pocas experiencias en las cuales los estudiantes utilizan herramientas colaborativas (como por ejemplo Wikis, google drive, etc.). Adicionalmente, los estudiantes cuentan con grupos de clase a través de los cuales pueden hacer consultas a sus compañeros respecto de dudas acerca de materias o instrucciones entregadas por los profesores, según explica uno de los entrevistados:

"Lo usamos por ejemplo si alguien no ha venido, para preguntar los deberes, o si alguien no le ha dado tiempo de copiar los deberes, o no sé, para hablar de cosas de esas".

En este sentido, se puede observar colaboración entre los estudiantes, pero siempre relacionada con la organización o gestión de información y no tanto para la elaboración colaborativa de productos.

c. Elaboración de productos: los estudiantes realizan sus trabajos escolares con el apoyo de las TIC. En particular, destaca el uso de software como procesadores de texto, programas de presentación y programas de edición. En menor medida y de acuerdo a las políticas de los centros educativos, algunos estudiantes también hacen uso de programas más específicos, sobre todo para desarrollar ejercicios de geometría o álgebra, según se pudo observar en la información registrada en los blogs. Al respecto, un resultado interesante de la investigación

Para efectos de esta investigación, el rendimiento académico está referido a las notas o evaluaciones obtenidas por los estudiantes.

3 Para efectos de esta investigación, el capital cultural está referido al interés y participación de los estudiantes en diferentes temáticas extraescolares. 
está relacionado con el rol de los centros educativos para optimizar la capacidad de beneficiarse del uso de las TIC. La mayoría de los entrevistados se sienten confiados en el uso de tecnología, han aprendido por sí solos lo que saben y creen que pueden aprender a utilizar cualquier software si se lo proponen. No obstante, aquellos estudiantes que provienen de centros educativos que cuentan con proyectos TIC, valoran mucho más la enseñanza asistida de ciertos programas, puesto que han aprendido con mayor rapidez programas que sus pares de otros institutos no conocen y cuentan con nuevas herramientas para sus actividades escolares y personales. En este sentido, es importante recalcar que la infraestructura tecnológica no basta para generar aprendizaje en los estudiantes, sino también la necesidad de contar con un proyecto educativo orientado a dar un uso pedagógico a las TIC.

\section{Promoción de malos hábitos educativos}

Los estudiantes reconocen que un uso inadecuado de las TIC puede dar paso a malos hábitos que va en desmedro de sus procesos de aprendizaje. En este sentido, reconocen que hay dos prácticas que son particularmente perjudiciales, a saber:

a. Abuso del copiado y pegado: los estudiantes se muestran críticos frente a los trabajos de investigación, porque indican que la mayoría de ellos no contribuye a su aprendizaje, puesto que se trata de buscar información, copiar lo encontrado (con pequeños cambios) y entregar el trabajo, sin que exista un proceso crítico o constructivo de la información. La mayoría de los estudiantes entrevistados coincide en indicar que usualmente consulta dos o tres sitios de Internet, copian lo que les parece más interesante y realizan pequeños arreglos de edición y cambios de palabras, tal como explica uno de los entrevistados:

"Lo que hago es que copio la información, la pego en un Word o en un Power Point o algo así, lo leo, miro si hay alguna cosa que no usaría yo, un tipo de vocabulario o algo, lo leo, intento de entender y si no lo entiendo, intentaría buscar otra cosa".

En algunos casos más aislados, el procedimiento es hacer esquemas y mapas conceptuales a partir de la información extraída desde Internet, para posteriormente hacer una redacción propia, pero estas iniciativas son escasas y propias de aquellos estudiantes sobresalientes o con mayor capital cultural. Al respecto, cabe destacar que no se observan diferencias en el proceso de búsqueda y adaptación de información según sexo de los estudiantes o titularidad del centro educativo. No obstante lo anterior, aquellos estudiantes que reconocen por parte de sus profesores condiciones más estrictas para realizar trabajos de investigación, evidencian mayor contribución de este tipo de estrategias pedagógicas, relevando nuevamente el rol de los centros educativos en los procesos de alfabetización digital de los estudiantes y su correspondiente capacidad de obtener provecho de las tecnologías.

b. Distracción en los estudios: los entrevistados reconocen que el uso de tecnología también es un distractor que les hace perder mucho tiempo para los estudios y para sus actividades cotidianas. Si bien las tecnologías permiten ahorrar tiempo, ese tiempo suele ser malgastado en el uso excesivo de juegos y redes sociales, distrayéndolos de las cosas realmente importantes. En dicho contexto, muchos de los nativos digitales se han visto obligados por sus padres a restringir el uso de las TIC. Dicha restricción obedece principalmente a dos razones, i) haber bajado su rendimiento académico, o ii) por la adicción en el uso de las TIC, advertida por los padres. A modo de castigo se les ha prohibido el uso total o parcial de las tecnologías hasta mejorar las notas o hasta que alcancen la capacidad de auto controlarse. Algunos estudiantes manifiestan que intentan evitar el uso de las TIC, llegando incluso a apagar el móvil o dejarlo en una habitación aparte, para evitar distracciones o pérdida de tiempo: 
"el móvil" lo tengo desconectado y fuera de mi alcance para que no me moleste, aunque a veces pienso ir al ordenador ${ }^{5}$ o ver el móvil, pero me suelo resistir".

En el ámbito personal de uso de las TIC, se pueden apreciar impactos independientes entre sí. Por un lado están los impactos asociados a las actividades de ocio y recreación de los estudiantes, esto es, cómo los estudiantes utilizan las TIC para actividades de entretenimiento, para organizar o buscar actividades de ocio o para el desarrollo de sus hobbies. Por otro lado se encuentran los impactos negativos, que están asociados principalmente a riesgos más que a efectos propiamente tal.

\section{Promoción de actividades de recreación y ocio}

De acuerdo a los nativos digitales, el uso de las TIC conlleva una serie de ventajas para su vida cotidiana. Plantean que pueden estar conectados e informados constantemente. Resaltan también el uso de las TIC como una herramienta que les facilita la búsqueda de información respecto de temas que son de su interés, lo que les permite aprender nuevos temas o adquirir nuevas habilidades. En particular, los impactos positivos son percibidos en tres áreas:

a. Comunicación permanente: alaban la instantaneidad y los bajos costos a través de los cuales pueden comunicarse, aludiendo a que pueden mantener una comunicación constante y fluida, con un gran número de personas a nivel nacional e internacional, sin incurrir en grandes gastos:

"sobre todo la comunicación instantánea, que cualquier cosa la puedes decir al instante, que puedes hablar con personas de diferentes lugares sin tener que pagar...”.

Cuentan con un perfil en las redes sociales más populares (Tuenti, Facebook, Twitter e Instagram), sin embargo, declaran que mantienen una actitud pasiva, dedicada principalmente a seguir noticias, informarse de lo que sucede con personas con las cuales han dejado de tener contacto, ver las publicaciones o fotografías de amigos y, eventualmente, darles un "me gusta". Con todo, algunos nativos digitales presentan un perfil un poco más activo, realizando algún tipo de publicación referida a su estado emocional, alguna frase que los identifique, alguna actividad cotidiana o subiendo fotos. Si bien es poco frecuente que publiquen acerca de temas relacionados con problemáticas globales, protestas sociales u otras temáticas de interés, algunos de los estudiantes sí valoran tener acceso a dichas publicaciones y poder informarse y mantenerse al tanto de lo que sucede en los temas que son de interés para ellos,

"...no es que participe, pero me hace abrir los ojos y darme cuenta de lo que realmente sucede".

b. Mayor acceso a actividades de ocio: los estudiantes manifiestan que las TIC facilitan la difusión de una serie de actividades de ocio y recreación que no cuentan con publicidad de pago, ampliando el abanico de posibilidades de actividades culturales, tales como eventos de cine, festivales, recitales, etc. Destacan particularmente el uso de redes sociales y páginas Web como promotoras de actividades emergentes y gratuitas, en su mayoría:

"es que puedo ver cine japonés, que no está en las salas de cine o me entero de encuentros de anime, cómics o qué sé yo....cosas de las que quizás no me enteraría si no fuese por Internet”.

c. Desarrollo de hobbies y temas de interés: los entrevistados plantean que las TIC les ayudan

Referido al teléfono móvil o teléfono celular

Referido al computador o computadora 
en el desarrollo de sus hobbies o pasatiempos. Si bien los jóvenes reconocen que muchas de las actividades podrían hacerlas sin ayuda de tecnología, manifiestan que las herramientas existentes en Internet les facilitan el acceso a la información, comunicación con pares, desarrollo de técnicas etc. Al respecto, una de las entrevistadas relata su experiencia en referencia a sus hobbies relacionados con el diseño de vestuario:

"Puedes encontrar diferentes técnicas, porque por ejemplo también tengo libros de costura, pero creo que en Internet tienes mucha más variedad de las técnicas que puedes encontrar". Adicionalmente, las TIC permite a los jóvenes ver y compartir experiencias con personas de distintos lugares geográficos, aumentando sus aprendizajes en áreas de interés, tal como se verá en la siguiente sección de resultados.

\section{Exposición a riesgos digitales}

Los estudiantes manifiestan que el uso excesivo o descuidado de las tecnologías puede traer consigo una serie de riesgos. En este sentido, los impactos negativos están asociados más a conductas de riesgos que a efectos o resultados propiamente tal. En concreto, los estudiantes advierten dos tipos de riesgos digitales:

a. Adicción digital: reconocen que amigos, pares o incluso ellos mismos son o han sido adictos al uso de tecnología, sintiendo una necesidad constante de usar el computador o el móvil, para jugar o conversar, y muchas veces privilegian esta actividad por sobre salir y compartir con amigos, tal como explica un entrevistado:

"Llegué a tener un poco de adicción a todo esto del ordenador y los videojuegos, y mis padres tuvieron que obligarme a no usar ordenador...".

Agregan que una de las desventajas del uso de la tecnología es la pérdida de tiempo. Si bien las tecnologías permiten ahorrar tiempo, ese tiempo suele ser malgastado en juegos y redes sociales, distrayéndolos de otras actividades, como las de interacción cara a cara:

"La gente se centra mucho más en la tecnología que en otras cosas, por ejemplo: la play, yo eso lo veo muy de —no salgo, porque estoy jugando a la play-”.

b. Riesgo de ciberbullying y vulnerabilidad de datos: los nativos digitales dan cuenta de la fragilidad de la privacidad y resguardo de datos en las redes sociales. Algunos de ellos reconocen que podrían ser víctimas de suplantación, robo o hackeo de cuentas. Además son conscientes de que las redes sociales pueden ser utilizadas para molestar, insultar o acosar a las personas, por conocidos o extraños. Con todo, las medidas de precaución de los nativos digitales hacia este tipo de riesgos varía dependiendo de sus experiencias al respecto. Si bien todos reconocen que el uso de las TIC está asociado a riesgos, la preocupación por ellos y la forma de enfrentar o disminuir la posibilidad de que ocurran depende de las experiencias previas que hayan tenido los jóvenes. A mayor experiencia con situaciones riesgosas en el uso de tecnología, mayor la preocupación por evitar riesgos, tal como se observa en la siguiente cita:

"es que yo hablo con gente que le conozco, al principio tenía muchos amigos, pero hace tiempo tuve un problema, por eso yo los elimine a todos y solo tengo personas que le conozco o que tengo confianza para tenerlos".

Como conclusión preliminar, se puede destacar que los impactos positivos no son transversales a todos los estudiantes entrevistados, observándose una relación entre el rendimiento académico de los estudiantes y la capacidad de obtener beneficios del uso de tecnologías, lo cual es particularmente evidente en los impactos referidos a los procesos educativos, y en especial en el referido al repaso de conceptos y contenidos. Situación 
similar a la que se observa en los impactos referidos al área personal, tales como mayor acceso a actividades de ocio y desarrollo de hobbies o temas de interés, en los cuales el capital cultural de los estudiantes es fundamental para entender su comportamiento con respecto a las TIC.

La capacidad de elaborar productos escolares, por su parte, está condicionada principalmente por la presencia de proyectos educativos con TIC en los centros educativos, o con la capacidad de los profesores de entregar instrucciones que orienten la elaboración de dichos productos. En este sentido, la posibilidad de lograr un provecho del uso de las TIC en el hogar, aumenta dependiendo de las políticas o prácticas pedagógicas de los centros educativos.

\subsection{APRENDIZAJES LOGRADOS A TRAVÉS DEL USO DE LAS TIC EN EL HOGAR}

Las TIC son utilizadas como un entorno de aprendizaje informal por los estudiantes, esto implica que los jóvenes desarrollan sus hobbies o talentos apoyándose en el uso de tecnologías, lo cual contribuye a mejorar sus aprendizajes en dichas áreas.

En particular, los temas o intereses de los entrevistados han sido clasificados en cinco grandes áreas de aprendizaje, los cuales son potenciados por herramientas tecnológicas para i) buscar información acerca del área de interés, ii) realizar actividades prácticas o iii) compartir y/o comunicar los productos derivados del área de interés, según se puede observar en la Tabla 2. De acuerdo a lo expuesto, en todas las áreas de aprendizaje, los jóvenes utilizan las tecnologías para leer o buscar información. Por el contrario, comunicar o compartir productos elaborados por los propios jóvenes, es una actividad que solo se observa en aquellos que muestran interés por las artes visuales o escénicas.

Tabla 2. Objetivo de uso de las TIC según área de aprendizaje

\begin{tabular}{|l|c|c|c|}
\hline \multirow{2}{*}{\multicolumn{2}{c|}{ Áreas de aprendizaje }} & \multicolumn{3}{c|}{ Objetivos del uso de TIC } \\
\cline { 2 - 4 } & $\begin{array}{c}\text { Leer e } \\
\text { Informarse }\end{array}$ & $\begin{array}{c}\text { Practicar o } \\
\text { aplicar }\end{array}$ & $\begin{array}{c}\text { Comunicar o } \\
\text { compartir }\end{array}$ \\
\hline Deportes & $\mathrm{x}$ & $\mathrm{x}$ & \\
\hline Idioma & $\mathrm{x}$ & $\mathrm{x}$ & $\mathrm{x}$ \\
\hline Artes escénicas y musicales & $\mathrm{x}$ & $\mathrm{x}$ & $\mathrm{x}$ \\
\hline Artes visuales, cine y fotografía & $\mathrm{x}$ & $\mathrm{x}$ & \\
\hline Historia y cultura & $\mathrm{x}$ & \\
\hline
\end{tabular}

Fuente. Elaboración propia

\section{Deportes}

Los procesos de aprendizaje en esta área de interés están relacionados con consultas acerca de reglas de juegos, técnicas para mejorar las prácticas deportivas, información 
acerca de los deportistas más destacados o de los lugares en donde es más recomendable el ejercicio del deporte (en el caso de natación, buceo, surfing, etc.). Adicionalmente, Internet suele ser utilizada para cotizar o comprar implementos, utilizar aplicaciones para registrar su actividad (por ejemplo kilómetros recorridos), etc. Uno de los usos más específicos de las TIC respecto al deporte es el de aplicaciones afines, que permiten a los jóvenes registrar la actividad deportiva y compartirla con pares, tal como describe una de las jóvenes entrevistadas acerca de su experiencia practicando buceo:

"Yo llevo un ordenador que es como un reloj, entonces ahí te marca, pues la profundidad máxima, la temperatura, cosas así y entonces luego, con una aplicación del móvil, te da la opción de poner la temperatura o el traje que has llevado, la profundidad, donde has ido, que has visto, no sé...".

\section{Idioma}

Los estudiantes manifiestan que ven en las TIC una herramienta fundamental para el aprendizaje de idiomas, puesto que pueden ver series o películas en el idioma de su interés, hacer cursos en línea (gratis o de pago) o contactarse con extranjeros y mantener una conversación con ellos a través de redes sociales:

“...inglés, en el colegio no aprendía mucho, pero en el ordenador me metía a redes sociales en inglés y entonces de ahí sí que acabé aprendiendo mucho inglés...y luego francés, tengo unos amigos de mis padres que son canadienses de la zona francófona, entonces yo hablo con ellos por correo en francés... y por internet también aprendí algo de francés".

III. Artes escénicas y musicales

El uso de Internet en esta área está orientado principalmente a buscar información respecto de los principales expositores, técnicas o estilos de artes escénicas o musicales. Adicionalmente, utilizan internet para buscar videos o tutoriales respecto de coreografías que puedan replicar, acordes para aprender a tocar una canción, etc., según explica una de las entrevistadas:

"buscamos ideas de coreografía en Youtube, luego la grabamos y la publicamos en algún grupo cerrado, para practicar en casa, para que no se nos olviden los pasos...”.

No obstante lo anterior, el aprendizaje o las actividades realizadas en esta área rara vez son compartidas o publicadas en Internet.

\section{Artes visuales, cine y fotografía}

Los estudiantes utilizan Internet para buscar información respecto de técnicas de diseño, edición, fotografía, pintura, etc., nuevas tendencias, estrenos de películas, consultar opiniones acerca de su trabajo, etc. En el caso particular de la fotografía, los estudiantes manifiestan utilizar programas en línea o en el computador para mejorar y difundir sus trabajos. En términos generales, quienes se muestran interesados en realizar alguna de las actividades relacionadas con esta área, son quienes presentan mayor tendencia a compartir su trabajo. 
“....A veces las publico en Facebook o Instagram, o si no también si hay concursos, de haz una foto o publica una foto de tal cosa y podrás participar en tal concurso, también subo fotos de eso".

\section{Historia y cultura}

En el caso de los estudiantes interesados por temas históricos o culturales, las TIC son utilizadas para ver películas, documentales o recolectar información que les enseñe acerca de aquellas culturas o momentos históricos que son de su interés:

"Una de las formas en que adquirí todo este conocimiento y el medio por el cual he tenido acceso a todo este mundillo (cultura japonesa) ha sido la informática, el ordenador en general y toda la animación, animé, etc., está en la red, así que es mi principal fuente de acceso a esto".

En coherencia con lo anterior, se puede establecer que independiente del área de interés de los estudiantes, la búsqueda de información en páginas de Internet es transversal como un primer proceso de acercamiento. Los videos o tutoriales disponibles en Internet también son utilizados como medio de información y aprendizaje en todas las áreas, salvo deportes. Por el contrario, el uso de cursos en línea es la herramienta menos utilizada, aplicada únicamente a temas relacionados con idiomas, según se puede observar en la Tabla 3.

Tabla 3. Uso de herramientas TIC según área de aprendizaje

\begin{tabular}{|l|c|c|c|c|c|c|}
\hline \multirow{2}{*}{ Áreas de aprendizaje } & \multicolumn{6}{|c|}{ Uso de herramientas TIC } \\
\cline { 2 - 7 } & $\begin{array}{c}\text { Blog y } \\
\text { foros }\end{array}$ & $\begin{array}{c}\text { Videos y } \\
\text { tutoriales }\end{array}$ & $\begin{array}{c}\text { Páginas de } \\
\text { búsqueda }\end{array}$ & $\begin{array}{c}\text { Cursos } \\
\text { en línea }\end{array}$ & $\begin{array}{c}\text { Programas y } \\
\text { aplicaciones }\end{array}$ & $\begin{array}{c}\text { Redes } \\
\text { sociales }\end{array}$ \\
\hline Deportes & & & $\mathrm{x}$ & $\mathrm{x}$ & $\mathrm{x}$ & $\mathrm{x}$ \\
\hline Idioma & & $\mathrm{x}$ & $\mathrm{x}$ & $\mathrm{x}$ & & $\mathrm{x}$ \\
\hline $\begin{array}{l}\text { Artes escénicas } \\
\mathrm{y} \text { musicales }\end{array}$ & $\mathrm{x}$ & $\mathrm{x}$ & $\mathrm{x}$ & & $\mathrm{x}$ & $\mathrm{x}$ \\
\hline $\begin{array}{l}\text { Artes visuales, } \\
\text { cine y fotografía }\end{array}$ & $\mathrm{x}$ & $\mathrm{x}$ & $\mathrm{x}$ & & & \\
\hline Historia y cultura & & & & & & \\
\hline
\end{tabular}

Fuente. Elaboración propia

Adicionalmente y tal como sucede con los impactos del uso de las TIC en el hogar, se observa una relación entre el rendimiento académico de los estudiantes y el capital cultural de estos en los procesos de búsqueda de información, dado que no todos los estudiantes tienen hobbies o temas de interés a los cuales destinen tiempo y dedicación. 


\section{CONCLUSIONES Y DISCUSIÓN}

De acuerdo a los resultados obtenidos, se puede observar que el uso de las TIC por parte de los estudiantes de secundaria responde a objetivos personales y educativos, reconociendo principalmente tres tipos de actividades: comunicación, entretenimiento y búsqueda de información, tal como ha sido advertido en investigaciones anteriores (Heim et al., 2007; Hinostroza et al., 2014; Peter y Valkenburg, 2006).

A partir de dichas actividades, algunos estudiantes logran obtener resultados positivos, asociados principalmente a los procesos de aprendizaje formal e informal. Estos hallazgos serían coincidentes con aquellos que demuestran que el uso de las TIC en el hogar puede generar impactos positivos en el aprendizaje y rendimiento académico de los estudiantes (Beltran et al., 2008; Chowdry et al., 2009; OECD, 2010).

En efecto, los resultados de la investigación demuestran que los estudiantes han logrado aprender o desarrollar diferentes áreas de interés (artes, música, idioma, etc.) gracias al apoyo de TIC, utilizando estas herramientas principalmente para la búsqueda de información y ejercitar o practicar habilidades. No obstante, los estudiantes son poco propensos a comunicar o publicar sus producciones o logros, resultados que desafían los planteamientos de quienes postulan que los nativos digitales, como colectividad, son una generación tendiente a publicar y compartir sus proyectos, opiniones, ideas o producciones propias (Prensky, 2001; Tapscott, 1998), demostrando un uso pasivo por sobre uno creativo (Selwyn, 2009).

Adicionalmente, se observa que son principalmente los estudiantes con alto capital cultural (Bourdieu, 1997) quienes tienen mayor capacidad de adquirir nuevos conocimientos y desarrollar sus hobbies a través del uso de las TIC, con lo cual es importante indagar con mayor detención en estrategias que permitan obtener un uso significativo de las TIC en un mayor número de estudiantes, reduciendo las brechas de usos (Heeks, 2008; Selwyn, 2004). En este sentido, y tal como plantean Castañeda y Adell (2014), es necesario realizar investigaciones orientadas a comprender los procesos que subyacen a estructurar los aprendizajes informales de los estudiantes a través del uso de tecnologías, centrándose en las actividades y procesos que estructuran los entornos personales de aprendizajes.

Por otro lado, se observan algunos impactos negativos relacionados con los hábitos de uso de las TIC, los cuales van en desmedro de sus procesos de aprendizaje formal, esto es particularmente evidente en lo referido a las prácticas de búsqueda de información para trabajos escolares, tal como han adelantado otros investigadores (Bennett et al., 2008; Cabra y Marciales, 2009; Combes, 2008), prácticas que se basan en la consulta de los dos o tres primeros sitios arrojados por el buscador, herramienta en la cual confían ciegamente, tal como plantea Selwyn (2009) y Combes (2009).

En coherencia, es importante llamar la atención, respecto de la necesidad de incorporar a los programas educativos materias de alfabetización digital que incluyan habilidades de búsqueda de información (Cabra y Marciales, 2009; Selwyn, 2009), puesto que se observó que aquellos estudiantes que se encuentran en centros educativos con proyectos de informática educativa obtienen más provecho del uso de las tecnologías que quienes no. En este contexto, es importante recalcar que las TIC deben ser consideradas como una palanca de cambio que debe ser utilizada intencionalmente para producir cambios (Venezky, 2002), y no esperar impactos per se, con lo cual los programas educativos debiesen avanzar en el desarrollo de estrategias educativas que desarrollen en los estudiantes un uso significativo, critico, ético y productivo de las TIC. 
Adicionalmente, los resultados desafían las creencias de las cualidades multitarea de los jóvenes (García, et al., 2007; Greenfield, 1985; Pedró, 2006; Prensky, 2001; Tapscott, 1998), toda vez que son ellos mismos quienes reconocen la distracción que generan las TIC a la hora de estudiar, reafirmando la postura de los investigadores que plantean que la multitarea es un mito (Reig, 2013; Rosen, 2008).

Si bien esta investigación ha entregado algunas luces respecto de los impactos o efectos que se pueden obtener del uso de las TIC en el hogar, es importante considerar que se trata de un estudio exploratorio con una muestra pequeña, con lo cual es necesario seguir profundizando en el tema a partir de investigaciones de mayor alcance.

\section{REFERENCIAS BIBLIOGRÁFICAS}

Adell, J., \& Castañeda, L. (2010). Los entornos personales de aprendizaje (PLEs): una nueva manera de entender el aprendizaje. In R. Roig \& M. Fiorucci (Eds.), Claves para la investigación en innovación y calidad educativas. La integración de las Tecnologías de la Información y la comunicación y la interculturalidad en las aulas. Roma: TRE Universita degli studi.

Andréu, J., García-Nieto, A., \& Pérez, A. (2007). Evolución de la Teoría Fundamentada como técnica de análisis cualitativo. Madrid: CIS.

Balanskat, A., \& Blamire, R. (2007). ICT in Schools: Trends, innovations and issues in 20062007. European Schoolnet. Recuperado desde http://insight.eun.org/shared/data/pdf/ict_in_ schools_2006-7_final.pdf

Beltran, D., Das, K., \& Fairlie, R. (2008). Are Computers Good for Children? The Effects of Home Computers on Educational Outcomes (Discussion Paper. Vol. 576). Australia: Centre for Economic Policy Research.

Bennett, S., Maton, K., \& Kervin, L. (2008). The digital natives debate: A Critical Review of the evidence. British journal of educational technology, 39(5), 775-786.

Bourdieu, P. (1997). Capital cultural, escuela y espacio social. México: Siglo XXI.

Bruns, A. (2007). Produsage: Towards a broader framework for user-led content creation. Artículo presentado en the 6th ACM SIGCHI conference on Creativity \& Cognition, Washington, DC.

Cabra, F., \& Marciales, G. (2009). Nativos digitales: ¿Ocultamiento de factores generadores de fracaso escolar? Revista Iberoamericana de educación, 50, 113-130.

Carrero, V., Soriano, R., \& Trinidad, A. (2012). Teoría Fundamentada, el desarrollo de teoría desde la generalización conceptual. Madrid: CIS.

Carstens, A., \& Beck, J. (2005). Get Ready for the gamer generation. Tech Trends, 49(3), 22-25.

Cassany, D., \& Ayala, G. (2008). Nativos e inmigrantes digitales en la escuela. CEE Participación Educativa, 9, 53-71.

Castañeda, L., \& Adell, J. (2014). Beyond the tools: analysing personal and group learning environments in a university course/ Más allá de la tecnología: análisis de los entornos de aprendizaje personales y grupales de estudiantes en una asignatura universitaria. Cultura y educación: Cultura and Education, 26(4), 739-774. doi:10.1080/11356405.2014.985946

Chiappe, A., Mesa, N. C., \& Álvarez, C. Y. (2013). Transformaciones en las concepciones de los docentes de educación secundaria acerca de la Web 2.0 y su uso en los procesos de enseñanza. Estudios Pedagógicos, XXXIX(2), 55-66.

Chowdry, H., Crawford, C., \& Goodman, A. (2009). Drivers and Barries to Educational Success. Evidence from the Longitudinal Study of young People in England. London: Institute for Fiscal Studies/DCSF.

Claro, M. (2010). Impacto de las TIC en los aprendizajes de los estudiantes. Estado del arte. Documento de Proyecto. 
Coll, C., Engel, A., Saz, A., \& Bustos, A. (2014). Personal learning environments: design an use/ Los entornos personales de aprendizaje en la educación superior: del diseño al uso. Cultura y educación: Cultura and Education, 26, 775-801. doi:10.1080/11356405.2014.985935

Coll, C., Mauri, T., \& Onrubia, J. (2008a). Análisis de los usos reales de las TIC en contextos educativos formales: una aproximación socio-cultural. Revista Electrónica de Investigación Educativa, 10(1), 1-18.

Coll, C., Mauri, T., \& Onrubia, J. (2008b). La utilización de las tecnologías de la información y la comunicación en la educación: Del diseño tecno-pedagógico a las prácticas de uso. In C. Coll \& C. Monereo (Eds.), Psicología de la educación virtual. Aprender y enseñar con las Tecnologías de la Información y la Comunicación. Madrid: Ediciones Morata.

Combes, B. (2008). The net generation: Tech-savvy or lost in virtual space. Paper presented at the IASL Conference: World class learning and literacy through school libraries, Berkeley.

Combes, B. (2009). Digital natives or digital refugees? Why we have failed gen Y? Artículo presentado en 38th Annual Conference of the International Association of School Librarianship: incorporating the 13th International Forum on Research in School Librarianship: Preparing pupils and students for the future, school libraries in the picture. Abano Terme.

Condie, R., \& Munro, B. (2007). The impact of ICT in schools. UK: Becta.

Cox, M., \& Marshall, G. (2007). Effects of ICT: Do we know what we should know? Education and Information Technologies, 12(2), 59-70. doi:10.1007/s10639-007-9032-х

Cuban, L. (2003). So much high-tech money invested, so little use and change in practice: how come? Retrieved from http://www.faculty.pnc.edu/dpratt/271/cuban.htm website

Demetriadis, S., Barbas, A., Molohides, A., Palaigeorgiou, G., Psillos, D., Vlahavas, I., . . Pombortsis, A. (2003). "Cultures in negotiation": teachers' acceptance/resistance attitudes considering the infusion of technology into schools. Computer \& Education, 41, 19-37.

Ertmer, P., Addison, P., Lane, M., Ross, E., \& Woods, D. (1999). Examining teachers' beliefs about the role of technology in the elementary classroom. Journal of Research on computing in Educaction, 32(1), 54-71.

Espinar, E., \& González, M. J. (2008). Jóvenes conectados. Las Experiencias de los jóvenes con las nuevas tecnologías. Revista Española de Sociología, 9, 109-122.

García, A., López-de-Ayala, M. C., \& Catalina, B. (2013). Hábitos de uso en Internet y en las redes sociales de los adolescentes españoles. Comunicar, XXI(41), 195-204. doi: 10.3916/C41-2013-19

García, F., Portillo, J., Romo, J., \& Benito, M. (2007). Nativos digitales y modelos de aprendizaje. Artículo presentado en IV Simposio Pluridisciplinar sobre Diseño, Evaluación y Desarrollo de Contenidos Educativos Reutilizables (SPDECE07), Bilbao.

García, F., \& Rosado, M. (2012). Conductas sociocomunicativas de los nativos digitales y los jóvenes en la web 2.0. Communication \& Society/Comunicación y Sociedad, 25(1), 15-38.

Glaser, B., \& Strauss, A. (1974). The Discovery of Grounded Theory, Strategies for qualitative research. Chicago: Aldine Publishing Company.

Gorden, R. L. (1969). Interviewing: strategy, techniques, and tactics. Homewood, III: Dorsey Press.

Gordo, Á. (2006). Jóvenes y cultura messenger. Madrid: Injuve.

Greenfield, M. (1985). El niño y los medios de comunicación. Madrid: Ediciones Morata.

Gu, X., Zhu, Y., \& Guo, X. (2013). Meeting the "Digital Natives": Understanding the Acceptance of Technology in Classrooms. Educational Technology \& Society, 16(1), 392-402.

Heeks, R. (2008). ICT4D 2.0: The Next Phase of Applying ICT for International Development. Computer, 41(6), 26-33.

Heim, J., Brandtzaeg, P. B., Hertzberg, B., Endestad, T., \& Torgersen, L. (2007). Children's usage of media technologies and psychosocial factors. New Media \& Society, 9(3), 425-454. doi: $10.1177 / 1461444807076971$

Hinostroza, E., Labbé, C., Brun, M., \& Matamala, C. (2011). Teaching and learning activities in Chilean classrooms: Is ICT making a difference? Computer \& Education, 57, 1358-1367. 
Hinostroza, E., Matamala, C., Labbé, C., Claro, M., \& Cabello, T. (2014). Factor (not) affecting what students do with computers and internet at home. Learning, Media and Technology, (ahead-ofprint), 1-21. doi:10.1080/17439884.2014.883407

Hood, J. (2010). Orthodoxy vs. Power: The Defining Traits of Grounded Theory. In A. Bryant \& K. Charmaz (Eds.), The SAGE Handbook of Grounded Theory. London: SAGE Publications Ltd.

Howe, N., \& Strauss, W. (2000). Millennials rising: the next great generation. New York: Vintage.

Hull, R. (2011). The Digital Native Are the Entertainment! EContent. Retrieved from http:// www.econtentmag.com/Articles/Column/Screen-Play/The-Digital-Natives-Are-theEntertainment!-77335.htm website

Keen, A. (2007). Cult of the amateur. New York: Random House.

Kennedy, G., Judd, T., Churchward, A., \& Gray, K. (2008). First year students' experiences with technology: Are they really digital natives? Australasian Journal of Educational Technology, 24(1), 108-122.

Kennedy, G., Judd, T., Dalgarnot, B., \& Waycott, J. (2010). Beyond natives and inmigrants: exploring types of net generation students. Journal of Computer Assisted Learning, 26, 332-343.

Kulik, J. (2003). Effects of Using Instructional Technology in Elementary and Secondary Schools: What Controlled Evaluation Studies Say. Arlington: VA: SRI International

Livingstone, S. (2012). Critical reflections on the benefits of ICT in education. Oxford Review of Education, 38(1), 9-24.

Livingstone, S., Haddon, L., Gorzig, A., \& Olafsson, K. (2011). Risks and safety on the internet: The perspective of European children. Full Findings. London: EU Kids Online.

Oblinger, D. (2003). Boomers, Gen-Xers, Millennials. Understanding the new students. Educase Review, 500(4), 37-47.

OECD. (2010). Are the New Millennium Learners Making the grade? Technology use and educational performance in PISA. París: OECD.

OECD. (2011). Informe PISA 2009: Estudiantes en Internet: Tecnologías y rendimiento digitales (Santillana Ed.). Madrid: Santillana.

Oliver, B., \& Goerke, V. (2007). Australian undergraduates' use and ownership of emerging technologies: Implications and opportunities for creating engaging learning experiences for the Next Generation. Australasian Journal of Educational Technology, 23(2), 171-186.

Pavez, M. I. (2014). Los derechos de la infancia en la era de Internet, América Latina y las nuevas tecnologías. Santiago de Chile: Cepal, Unicef.

Pedró, F. (2006). Aprender en el nuevo milenio: Un desafío a nuestra visión de las tecnologías y la enseñanza. París: OECD-CERI.

Pedró, F. (2011). Tecnología y escuela: lo que funciona y por qué. Madrid: Fundación Santillana.

Pelgrum, W. J. (2001). Obstacles to the integration of ICT in education: results from a worldwide educational assessment. Computer \& Education, 37, 163-178.

Peter, J., \& Valkenburg, P. (2006). Adolescents' internet use: Testing the "disappearing digital divide" versus the "emerging digital differentiation" approach. Poetics, 34, 293-305. doi:10.1016/j. poetic.2006.05.005

Prensky, M. (2001). Nativos e Inmigrantes digitales. On the horizon, 9(5), 1-7. Ramboll

Management. (2006). E-learning Nordic 2006. Impact of ICT in Education. Copenhagen: Ramboll Management.

Reig, D. (2013). Describiendo al hiperindividuo, el nuevo individuo conectado. Los jóvenes en la era de la hiperconectividad: tendencias, claves y miradas (pp. 21-90). Madrid: Fundación Telefónica - Fundación Encuentro.

Robert, I. (2013). Digital Natives or Digital tribes. Universal Journal of Educational Research, 1(2), 104-112.

Roman, M. (2010). Cuatro formas de incorporar las TIC a la enseñanza en el aula. En A. Bilbao \& Á. Salinas (Eds.), El libro abierto de la informática educativa: Lecciones y desafíos de la Red 
Enlaces (pp. 105-122). Santiago: Enlaces, Centro de Educación y Tecnología del Ministerio de Educación.

Rosen, C. (2008). The Myth of Multitasking. The New Atlantis, 20, 105-110.

Ruiz, P. (2013). Nuevas tecnologías y estudiantes chilenos de secundaria. Aportes a la discusión sobre la existencia de nuevos aprendices. Estudios Pedagógicos, XXXIX(2), 279-298.

Sánchez, J., Salinas, A., Contreras, D., \& Meyer, E. (2011). Does the new digital generation of learners exist? A qualitative study. British journal of educational technology, 42(4), 543-556.

Selwyn, N. (1998). The effect of using a home computer on students' educational use of IT. Computer \& Education, 31, 211-227.

Selwyn, N. (2004). Reconsidering political and popular understandings of the digital divide. New Media \& Society, 6(3), 341-362. doi:10.1177/1461444804042519

Selwyn, N. (2009). The digital native - myth and reality. Aslib Proceedings: New Information Perspectives, 61(4), 364-379.

Strauss, A. (1987). Qualitative analysis for social scientists. Cambridge, UK: University Press.

Strauss, A., \& Corbin, J. (1990). Basic of Qualitative Research. California: Sage Publications.

Sunkel, G., Trucco, D., \& Moller, S. (2011). Aprender y enseñar con las tecnologías de la información y las comunicaciones en América Latina: potenciales beneficios. Santiago de Chile: CEPAL.

Tapscott, D. (1998). Creciendo en un entorno digital: La generación Net. Bogotá: Mc Graw Hill.

Thompson, P. (2013). The digital matives as learners: Technology use patterns and approaches to learning. Computer \& Education, 65, 12-33.

Valles, M. (1997). Técnicas cualitativas de investigación social, Reflexión metodológica y práctica profesional. Madrid: Editorial Síntesis.

Valles, M. (2002). Entrevistas cualitativas. Madrid: CIS.

Venezky, R. L. (2002, March 6). Quo Vademus? The transformation of schooling in a networked world. Retrieved from http://www.oecd.org/dataoecd/48/20/2073054.pdf

Williams, D., Crittenden, V., Keo, T., \& McCarty, P. (2012). The use of social media: an exploratoru study of usage among digital natives. Journal of Public Affairs, 12(2), 127-136.

Yong, S.-T., \& Gates, P. (2014). Born Digital: Are they really Digital Natives? International Journal of e-Education, e-Business, e-Management and e-Learning, 4(2), 102-105. doi:10.7763/ IJEEEE.2014.V4.311 
